\title{
Effects of Isulin-like Growth Factor-I on Maternal and Fetal Plasma Amino Acid Levels in Pregnant Rats
}

\author{
Boonrit THONGSONG ${ }^{1,2)}$, Makoto BONKOBARA ${ }^{2)}$, Mitsuhito MATSUMOTO ${ }^{2)}$, Jong-sik JANG ${ }^{2)}$, Naoaki MATSUKI ${ }^{2)}$, \\ Mutsumi INABA ${ }^{2)}$ and Kenichiro $\mathrm{ONO}^{2)}$ \\ ${ }^{1)}$ Faculty of Veterinary Science, Chulalongkorn University, Bangkok, Thailand and ${ }^{2)}$ Department of Veterinary Clinical Pathobiology, \\ Graduate School of Agricultural and Life Sciences, The University of Tokyo, 1-1-1 Yayoi, Bunkyo-ku, Tokyo 113-8657, Japan.
}

(Received 13 June 2000/Accepted 20 May 2002)

ABSTRACT. Pregnant rats were subcutaneously administered with recombinant human insulin-like growth factor-I (rhIGF-I) in doses of 0 (control), 1, 2, and $4 \mu \mathrm{g} / \mathrm{g}$ body weight per day from day 18 to 21 of pregnancy. On day 21 of pregnancy, maternal and fetal plasma samples were collected and those amino acid levels were measured. The ratios of fetal/maternal plasma amino acids, especially 1 eucine, isoleucine, tryptophan, phenylalanine and tyrosine, increased in $2 \mu \mathrm{g}$ rhIGF-I treated group. Both total fetal weight and total placental weight were also higher than those in the control group. These results suggested that IGF-I enhanced fetal growth by, as one of its possible mechanisms, promoting placental amino acid supplies from the mother to fetuses.

KEY WORDS: insulin-like growth factor-I (IGF-I), plasma amino acid, pregnant rat.

J. Vet. Med. Sci. 64(9): 859-861, 2002

Insulin-like growth factor-I (IGF-I) is widely known to be a major growth factor for many types of cells including placental cell [4] and also an important regulatory factor for fetal growth and development $[11,16]$. Fetal tissue and placenta expressed a large number of IGF receptor $[12,14]$. Gluckman et al. [6] demonstrated that the administration of recombinant human IGF-I (rhIGF-I) to pregnant rats enhanced fetal growth. In most species, maternal serum levels of IGF-I correlated positively with fetal and/or placental weight $[7,15]$. On the other hand, the supply of amino acids from the mother to fetuses via placenta is crucial importance for fetal growth. The full term fetal weight was reduced by the starvation at late phase of pregnancy with the decrease of fetal plasma amino acids level and also fetal/maternal plasma amino acids ratio in the rat [1]. Impaired fetal and placental uptake of amino acids has been observed in several models of growth retardation in the rat [8]. This note deals with the effects of IGF-I on maternal and fetal plasma amino acid concentrations in pregnant rats.

Timed pregnant Wister ST rats (weighing 250-270 g) were obtained from Japan SLC (Shizuoka, Japan) and divided into four treatment groups. The rats were housed in standardized cages with controlled temperature under a 12hr light-dark cycle and received standard rat chow and drinking water ad libitum. The rats were subcutaneously administered with rhIGF-I (Fujisawa Pharmaceutical Co., Ltd., Osaka, Japan) in doses of 0 (control), 1, 2, and $4 \mu \mathrm{g} / \mathrm{g}$ body weight per day in $0.6 \mathrm{~m} l$ of physiological saline, based on the previous reports $[2,5,6]$ and our preliminary study. The total of 6 administrations was given every $12 \mathrm{hr}$ to each rat starting from at 19:00 on day 18 of pregnancy. Three

\footnotetext{
* Correspondence to: Ono, K., Department of Clinical Pathobiology, Graduate School of Agricultural and Life Sciences, The University of Tokyo, Yayoi 1-1-1, Bunkyo-ku, Tokyo 1138657, Japan.
}

hours after the final administration on day 21, 1 day before delivery, the rats were anesthetized with diethyl ether. Then, blood samples collected from maternal artery were transferred into heparinized tubes on ice and centrifuged at $1,500 \times \mathrm{g}$ for $10 \mathrm{~min}$ at $4^{\circ} \mathrm{C}$. Plasma samples were separated and stored at $-20^{\circ} \mathrm{C}$ until assay. After blood sampling, individual placentas and fetuses were removed and weighed, and fetal blood samples were collected by the decapitation. Both total fetal weight and placental weight in $2 \mu \mathrm{g}$ treated group was heavier than those of the control group (Table 1). The rats were killed by blood withdrawal under diethyl ether.

Plasma amino acid levels were determined by a high-performance liquid chromatography (HPLC: LCSS-905, JASCO, Tokyo, Japan) with a pre-column dabsylation procedure [17]. Briefly, plasma samples were deproteinated by 4-fold volume of acetonitrile and centrifuged at $10,000 \times \mathrm{g}$ for $15 \mathrm{~min}$ at $4^{\circ} \mathrm{C}$. The supernatant was collected and dabsylated with a commercial kit (Amino-chrome analysis kit, Ciba-Corning, Essex, England). Then, dabs-amino acid derivatives were determined by a reversed phase HPLC system using an Amino-chrome C18 analytical column (CibaCorning, Essex, England) and Crest-pak C18 guard column (JASCO, Tokyo, Japan). A high-pressure gradient elution with two solvents was prepared; (A): $25 \mathrm{mM}$ sodium acetate, $4 \%$ N,N-dimethylformamide, $\mathrm{pH} 6.4$, and (B): acetonitrile. The gradient was formed as follows with (A)\%: $85 \%$ at $0 \mathrm{~min}, 70 \%$ at $31 \mathrm{~min}, 59 \%$ at $35 \mathrm{~min}, 46 \%$ at $39 \mathrm{~min}$, $43 \%$ at $47 \mathrm{~min}, 33 \%$ at $48 \mathrm{~min}, 10 \%$ at $49 \mathrm{~min}, 85 \%$ at 50 min with flow rate at $1.0 \mathrm{ml} / \mathrm{min}$. Twenty microliter of sample was applied and the eluate was monitored at the wave length of $465 \mathrm{~nm}$. Column temperature was kept at $40^{\circ} \mathrm{C}$ and total elution time was $50 \mathrm{~min}$. All data were analysed by student's $t$-test.

Table 2 shows maternal and fetal plasma levels of indi- 
Table 1. Changes of maternal and fetal body weight, and maternal feed consumption after the administration of rhIGF-I

\begin{tabular}{|c|c|c|c|c|}
\hline \multirow[b]{2}{*}{ Parameters } & \multirow[b]{2}{*}{$\begin{array}{c}\text { Control } \\
(\mathrm{n}=12)\end{array}$} & \multicolumn{3}{|c|}{ rhIGF-I doses ( $\mu \mathrm{g} / \mathrm{g}$ body weight/day) } \\
\hline & & $\begin{array}{c}1 \\
(\mathrm{n}=11)\end{array}$ & $\begin{array}{c}2 \\
(\mathrm{n}=12)\end{array}$ & $\begin{array}{c}4 \\
(n=11)\end{array}$ \\
\hline $\begin{array}{c}\text { Maternal empty weight }(\mathrm{g}) \\
\text { daily gain ( } \mathrm{g} / \text { day })\end{array}$ & $285.6 \pm 20.1$ & $279.1 \pm 24.0$ & $295.9 \pm 30.4$ & $288.4 \pm 26.4$ \\
\hline before & $7.49 \pm 2.04$ & $6.40 \pm 1.72$ & $7.83 \pm 2.13$ & $5.62 \pm 1.42$ \\
\hline after & $9.23 \pm 2.58$ & $11.76 \pm 2.28$ & $12.38 \pm 2.18 * *$ & $11.48 \pm 2.17$ \\
\hline Daily feed consumption $(\mathrm{g} /$ & & & & \\
\hline before & $24.56 \pm 3.56$ & $22.97 \pm 4.83$ & $23.01 \pm 4.74$ & $22.67 \pm 5.06$ \\
\hline after & $23.49 \pm 4.12$ & $22.27 \pm 3.12$ & $23.55 \pm 3.70$ & $23.02 \pm 3.88$ \\
\hline Total fetal weight $(\mathrm{g})$ & $39.90 \pm 5.85$ & $44.20 \pm 8.13$ & $46.92 \pm 5.72 * *$ & $40.58 \pm 5.93$ \\
\hline Total placental weight $(\mathrm{g})$ & $4.92 \pm 0.58$ & $5.47 \pm 1.35$ & $5.62 \pm 0.77 * *$ & $5.00 \pm 0.72$ \\
\hline Litter size (No.) & $11.4 \pm 2.0$ & $12.5 \pm 2.9$ & $13.00 \pm 1.5$ & $12.0 \pm 2.2$ \\
\hline
\end{tabular}

Values are means \pm SD. Number of animals are shown in parentheses, except maternal feed utilization efficiency $(n=4)$. Before administration; from day 15 to day 17 of pregnancy and after administration; from day 18 to day 21 of pregnancy. $* * \mathrm{P}<0.01$; significant difference vs. control group.

Table 2. Concentrations of individual amino acid $(\mu \mathrm{M})$ in maternal and fetal plasma after the administration of rhIGF-I

\begin{tabular}{|c|c|c|c|c|c|c|c|c|}
\hline \multirow{3}{*}{$\begin{array}{l}\text { Amino } \\
\text { acids }\end{array}$} & \multicolumn{4}{|c|}{ Maternal plasma } & \multicolumn{4}{|c|}{ Fetal plasma } \\
\hline & \multirow{2}{*}{$\begin{array}{c}\text { Control } \\
(\mathrm{n}=9)\end{array}$} & \multicolumn{3}{|c|}{ rhIGF-I ( $\mu \mathrm{g} / \mathrm{g}$ body weight/day) } & \multirow{2}{*}{$\begin{array}{l}\text { Control } \\
(\mathrm{n}=9)\end{array}$} & \multicolumn{3}{|c|}{ rhIGF-I ( $\mu \mathrm{g} / \mathrm{g}$ body weight/day) } \\
\hline & & $1(n=8)$ & $2(\mathrm{n}=9)$ & $4(n=8)$ & & $1(\mathrm{n}=8)$ & $2(\mathrm{n}=9)$ & $4(n=8)$ \\
\hline Glu & $44.42 \pm 19.71$ & $54.49 \pm 30.13$ & $39.48 \pm 16.38$ & $46.40 \pm 25.01$ & $297.36 \pm 95.92 \#$ & $261.23 \pm 76.98$ & $305.14 \pm 76.13$ & $286.28 \pm 60.88$ \\
\hline Asn & $28.09 \pm 11.78$ & $25.32 \pm 11.71$ & $1.38 \pm 8.60$ & $19.04 \pm 6.53$ & $100.37 \pm 41.25 \#$ & $95.83 \pm 30.47$ & $98.19 \pm 36.25$ & 5.16 \\
\hline Gln & $660.38 \pm 157.70$ & $602.02 \pm 167.34$ & $518.95 \pm 162.67$ & $493.85 \pm 144.10$ & $1870.23 \pm 521.15 \#$ & $1675.00 \pm 454.30$ & $1810.00 \pm 429.51$ & 1781.0 \\
\hline Ser & $98.86 \pm 24.65$ & $95.44 \pm 20.14$ & $76.30 \pm 19.56$ & $74.88 \pm 16.52$ & $247.72 \pm 68$ & $246.48 \pm 47.39$ & $251.80 \pm 56.07$ & 7.87 \\
\hline Thr & $217.91 \pm 41.91$ & $192.36 \pm 20.46$ & $155.17 \pm 48$ & $155.25 \pm 36.36^{* *}$ & $2.37 \pm 1$ & 513.22 & 69 & 0.64 \\
\hline Gly & $44.64 \pm 18.60$ & 50.08 & 4 & 4 & $.10^{-}$ & 211. & 00 & .28 \\
\hline Ala & $167.81 \pm 71.66$ & $145.48 \pm 62.92$ & $115.91 \pm$ & $55^{* *}$ & 96\# & 864.1 & 907.8 & 9.22 \\
\hline Arg & $44.82 \pm 28.91$ & $27.69 \pm 6.66$ & $65 \pm 9$ & 22.85 & $159.11 \pm$ & 151.28 & 163.8 & 7.09 \\
\hline Tau & $106.13 \pm 31.85$ & $105.88 \pm 35.78$ & $76.65 \pm 1$ & $87.62 \pm 14.97$ & $468.21 \pm$ & 429.43 & $443.08 \pm$ & 3.64 \\
\hline Pro & $107.76 \pm 22.47$ & $97.20 \pm$ & $84 \pm 31$ & 79.27 & $279.03 \pm$ & 252.79 & $298.33=$ & 275. \\
\hline Val & $118.42 \pm 18.94$ & $104.80 \pm$ & $85.43 \pm 14.60 * *$ & $90.09=$ & $5.71 \pm 8$ & $491.89 \pm 71.12$ & 500.34 & 494. \\
\hline Met & $30.39 \pm 10.98$ & $23.57 \pm 5.72$ & $18.33 \pm 6.96$ & $20.41 \pm$ & $103.90 \pm 17.99 \#$ & $99.03 \pm 12.97$ & $94.35 \pm 21.25$ & $106.01 \pm 17.85$ \\
\hline Ile & $74.02 \pm 23.22$ & $64.44 \pm 20.31$ & $46.95 \pm 15.84$ & $51.67 \pm 20.63$ & $321.71 \pm 51.86 \#$ & $326.88 \pm 47.61$ & $330.46 \pm 63.12$ & $317.48 \pm 65.77$ \\
\hline Leu & $133.16 \pm 27.32$ & $117.63 \pm 20.73$ & $97.64 \pm 12.79 * *$ & $100.11 \pm 26.19$ & $471.16 \pm 74.34 \#$ & $465.97 \pm 62.66$ & $461.29 \pm 71.15$ & $457.93 \pm 67.55$ \\
\hline Trp & $44.31 \pm 7.31$ & $37.33 \pm 6.51$ & $28.67 \pm 6.20^{* *}$ & $39.01 \pm 13.39$ & $100.44 \pm 23.51 \#$ & $108.66 \pm 21.66$ & $116.94 \pm 20.65$ & $116.70 \pm 27.72$ \\
\hline Phe & $56.27 \pm 8.58$ & $49.81 \pm 10.66$ & $42.86 \pm 6.79 * *$ & $45.37 \pm 10.99$ & $264.66 \pm 43.98 \#$ & $258.56 \pm 38.79$ & $285.01 \pm 47.67$ & $283.71 \pm 46.16$ \\
\hline Lys & $140.32 \pm 56.72$ & $159.96 \pm 29.14$ & $140.32 \pm 32.98$ & $139.36 \pm 42.29$ & $655.49 \pm 187.45 \#$ & $662.55 \pm 127.70$ & $713.82 \pm 210.99$ & $683.42 \pm 205.49$ \\
\hline His & $42.61 \pm 15.67$ & $30.53 \pm 8.13$ & $25.93 \pm 5.23 * *$ & $27.05 \pm 5.38$ & $144.55 \pm 54.12 \#$ & $133.48 \pm 37.05$ & $162.82 \pm 58.07$ & $139.62 \pm 46.70$ \\
\hline Tyr & $84.31 \pm 25.57$ & $61.36 \pm 19.66$ & $47.97 \pm 9.11 * *$ & $48.36 \pm 12.88 * *$ & $369.16 \pm 75.70 \#$ & $376.50 \pm 56.13$ & $409.03 \pm 88.95$ & $397.58 \pm 65.36$ \\
\hline
\end{tabular}

Number of animals are shown in parentheses. Values are mean \pm SD. \#; $\mathrm{P}<0.0001$, significant difference in fetus vs. mother in control group. **; $\mathrm{P}<0.01$, significant difference vs. control. Abbreviation: glutamic acid (Glu), asparagine (Asn), glutamine (Gln), serine (Ser), threonine (Thr), glycine (Gly), alanine (Ala), arginine (Arg), taurine (Tau), proline (Pro), valine (Val), methionine (Met), isoleucine (Ile), leucine (Leu), tryptophan (Trp), phenylalanine (Phe), lysine (Lys), histidine (His), tyrosine (Tyr).

vidual amino acids in the control and rhIGF-I treated groups. Those levels of mother and fetus in the control group, indicating that all amino acid level of fetal plasma were significantly higher $(\mathrm{P}<0.0001)$ than those of maternal plasma, were consistent with previous reports $[1,3,10]$. Maternal concentrations of 7 amino acids (threonine, valine, leucine, tryptophan, phenylalanine, histidine, and tyrosine) at $2 \mu \mathrm{g}$ and 3 amino acids (threonine, alanine, and tyrosine) at $4 \mu \mathrm{g}$ of rhIGF-I treated group were significantly lower $(\mathrm{P}<0.01)$ than those in the control group. In contrast, fetal plasma amino acid concentrations showed no significant changes over control. The ratios of fetal/maternal plasma amino acid concentrations were calculated as an index of placental amino acid transport activities $[1,13,18]$. The ratios of 6 amino acids (arginine, isoleucine, leucine, tryptophan, phenylalanine, and tyrosine) at $2 \mu \mathrm{g}$ and 2 amino acids (alanine and tyrosine) at $4 \mu \mathrm{g}$ of rhIGF-I treated groups were significantly higher $(\mathrm{P}<0.01)$ than those in the control.

Masuyama et al. [9] reported that IGF-I enhanced ami- 


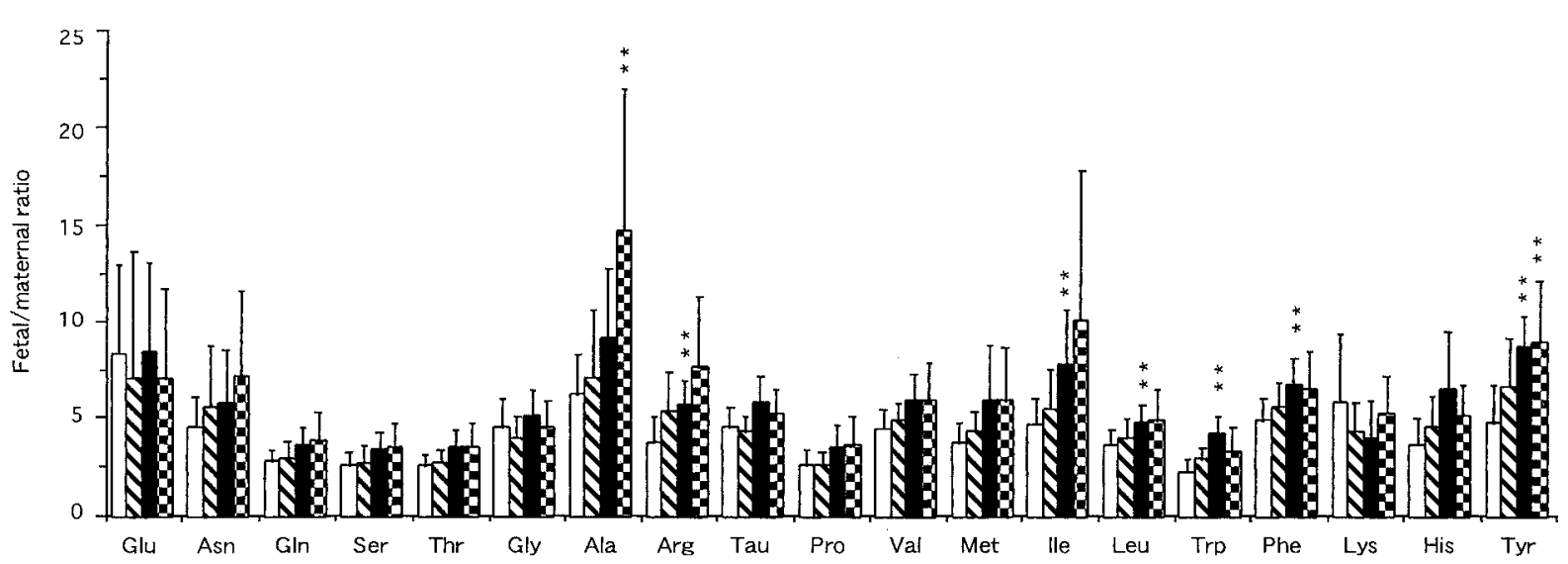

Fig. 1. The ratio of fetal to maternal

plasma amino acid concentrations after the administration of various dose $\quad$ control, $\mathrm{n}=9, \mathbb{R}: \mathrm{g} / \mathrm{g}$ body weight/day, $\mathrm{n}=8, \boldsymbol{\square}: 2 \mu \mathrm{g} /$ g body weight/day, $\mathrm{n}=9$, and $4 \mu \mathrm{g} / \mathrm{g}$ body weight/day, $\mathrm{n}=8$ ) of rhIGF-I. Values are means $\pm \mathrm{SD}$. $* * ; \mathrm{P}<0.01$, significant differences

noisobutyric acid (AIB), isoleucine, and alanine uptake by placental explant. In this study, the marked decrease of amino acid concentrations in maternal plasma was observed, when pregnant rats were administered with $2 \mu \mathrm{g}$ of rhIGF-I. Maternal plasma amino acid levels were kept low during pregnant period compared with those in the nonpregnant rat, probably because the enhancement of active amino acid transport from the mother to the fetus via placenta [13]. IGF-I might enhance some amino acid transfer by the placental uptake, by which decreased amino acid concentrations in the mother. The ratios of fetal/maternal amino acid concentration, suggesting placental transport activity, were higher at $2 \mu \mathrm{g}$ rhIGF-I treated group than in the control. In this study, fetal and placental weights increased at $2 \mu \mathrm{g}$ rhIGF-I treated group without changes of maternal body weight.

It has been widely accepted fetal plasma amino acid levels are remarkably elevated by the activated several amino acid transporters $[1,8]$. There are many factors and mechanisms that increase placental amino acids uptake. Our previous report demonstrated that epidermal growth factor (EGF) also changed circulating amino acid concentrations in dose-dependent manner associated with fetal and placental growth [3]. Further characterization of the specific amino acid transport activity is necessary to evaluate a major role of IGF-I on maternal and fetal plasma amino acids level and also fetal growth.

From these results, it was suggested that IGF-I enhanced fetal growth by, as one of its possible mechanism, promoting placental amino acid supplies from the mother to fetuses.

ACKNOWLEDGEMENT. The authors are grateful to the Fujisawa Pharmaceutical Co., Ltd, Osaka, Japan for the generous provision of rhIGF-I.

\section{REFERENCES}

1. Arola, LI., Palou, A., Remesar, X. and Alemany, M. 1982. Horm. Metabol. Res. 14: 364-371.

2. Bagi, CM., Deleon, E., Brommage, R., Adams, S., Rosen, D. and Sommer, A. 1995. Bone 16: 263s-269s.

3. Bonkobara, M., Thongsong, B., Onda, K., Matsuki, N., Inaba, M. and Ono, K. 1997. J. Vet. Med. Sci. 59: 1053-1056.

4. Fant, M., Munro, H. and Moses, AC. 1986. J. Clin. Endocrinol. Metab. 63: 499-505.

5. Gargosky, S.E., Owens, J.A., Walton, P.E., Owens, P.C., Wallace, J.C. and Ballard, F.J. 1991. Endocrinology 130: 395-400.

6. Gluckman, P.D., Morel, P.H.C., Ambler, G.R., Breier, B.H., Blair, H.T. and McCutcheon, S.N. 1992. Endocrinology 134: R1-R3.

7. Humbel, RE. 1990. Eur. J. Biochem. 190: 445-462.

8. Lewis, R.M., Bassett, N.S., Johnston, B.M. and Skinner, S.J.M. 1998. Placenta 19: 403-408.

9. Masuyama, H., Hiramatsu, Y. and Kudo, T. 1996. J. Perinat. Med. 24: 213-220.

10. McEvoy-Bowe, E., Hislop, J., Wiggins, D. and Lund, P. 1987. Bio. Neonate. 52: 135-140.

11. Morel, P.C.H., Blair, H.T., Ormsby, J.E., Breier, B.H., McCutcheon, S.N. and Gluckman, P.D. 1994. J. Reprod. Fertil. 101: 9-14.

12. Owens, P.C., Brinsmead, M.W., Waters, M.J. and Thorburn, D. 1980. Biochem. Biophys. Res. Commun. 96: 1812-1820.

13. Palou, A., Arola, L. and Alemany, M. 1977. Biochem. J. 166: 49-55.

14. Pilistine, S.J., Moses, A.C. and Munro, H.N. 1984. Endocrinology 115: 1060-1065.

15. Takeda, Y. and Iwashita, M. 1993. Ann. Acad. Med. 22: 134 141.

16. Unterman, T.G., Simmons, R.A., Glick, R.P. and Ogata, E.S. 1993. Endocrinology 132: 327-336.

17. Vendrell, J. and Aviles, F.X. 1986. J. Chromatogr. 358: 401413.

18. Yudilevich, D.L. and Sweiry, J.H. 1985. Biochem. Biophys. Acta. 822: 169-201. 